\title{
A Virtual Instrument Environment to Characterize Reverse Recovery Parameters in LDMOS Devices
}

\author{
Carlos J. Bernal and Manuel Jim'enez \\ Electrical and Computer Engineering Department \\ University of Puerto Rico, Mayag"uez \\ Mayag"uez, Puerto Rico 00681
}

\begin{abstract}
Measuring and quantifying reverse recovery parameters in high speed LDMOS devices is an important task in their characterization. A robust and automated procedure is required to achieve accurate and repeatable parameter quan-tification. This work focuses in the development of a virtual instrumentation setup to perform the reverse recovery parameter extraction in an automated manner. We define the architectural and functional requirements of such an environment to then introduce our proposed solution. The proposed design was developed in LabVIEWTMfor a low-cost instrument platform and its functionality assessed under several conditions to guarantee the consistency and repeatability of the measured data.
\end{abstract}

Digital Object Identifier (DOI):

http://dx.doi.org/10.18687/LACCEI2016.1.1.346

ISBN: 978-0-9822896-9-3

ISSN: 2414-6390

$14^{\text {th }}$ LACCEI International Multi-Conference for Engineering, Education, and Technology: "Engineering Innovations for Global Sustainability", 20-22 July 2016, San José, Costa Rica. 


\title{
A Virtual Instrument Environment to Characterize Reverse Recovery Parameters in LDMOS Devices
}

\author{
Carlos J. Bernal and Manuel Jiménez \\ Electrical and Computer Engineering Department \\ University of Puerto Rico, Mayagüez \\ Mayagüez, Puerto Rico 00681
}

\begin{abstract}
Measuring and quantifying reverse recovery parameters in high speed LDMOS devices is an important task in their characterization. A robust and automated procedure is required to achieve accurate and repeatable parameter quantification. This work focuses in the development of a virtual instrumentation setup to perform the reverse recovery parameter extraction in an automated manner. We define the architectural and functional requirements of such an environment to then introduce our proposed solution. The proposed design was developed in LabVIEW ${ }^{\mathrm{TM}}$ for a low-cost instrument platform and its functionality assessed under several conditions to guarantee the consistency and repeatability of the measured data.
\end{abstract}

\section{INTRODUCTION}

Laterally-Diffused MOSFETs (LDMOS) are fabricated through a CMOS process that creates a double diffusion on the device structure [1]. This double diffusion enables the usage of such devices in diverse power electronics and radio frequency applications [2].

A distinctive characteristic of LDMOS transistors is an intrinsic P-N junction formed between the device's source and drain regions due to their double diffusion. This junction, known as the reverse diode [3], produces a reverse current flow for a short period of time when the device is switched from forward to reverse. Fig 1 illustrates the behavior of this diode in what is called the device's reverse recovery process.

Determining the parameters governing the reverse recovery process is a vital task in LDMOS characterization. The parameters of interest include the reverse recovery current $\left(I_{r r}\right)$, storage charge $\left(Q_{r r}\right)$, reverse recovery time $\left(t_{r r}\right)$, storagecharge time $\left(t_{s}\right)$, reverse decay time $\left(t_{d}\right)$, and switching speed (di/dt) [4]. $Q_{r r}$ and $t_{r r}$ are needed to estimate the devices power losses and maximum switching frequency [5].

Performing an accurate and quick quantification of the Reverse Recovery Parameters (RRP) becomes a challenge, particularly when the task is meant to be performed in a production environment, where thousands of devices need to be tested. For this reason an automated procedure is required.

Completing a single test requires dealing with multiple tasks, that include instruments setup and communications protocols, test architecture, DUT excitation, and data acquisition and analysis, among others. The cost of the test equipment used in the procedure is also a relevant factor to be considered when dealing with the characterization process.
Several works in the recent literature have dealt with the problem of automating RRT characterization in diverse types of devices. D. Kuebrich et Al. developed an automated system to determine the reverse recovery behavior of fast diodes [6]. They proposed driving all instruments in the test with an ATmega 16 microcontroller (MCU). They developed a communications interface via MATLAB with a personal computer for the main control. They used the MCU to handle the application of the reverse voltage, the control pulse, and controlling the measurement instruments. All configuration parameters were entered via MATLAB. They did not explain how they implemented the post processing stage required to quantify the computed RRPs. Although their system was able obtain the desired parameters, the test could not be performed in real time due to features of their software.

J. Rodríguez et $A l$. reported using used $C \sharp$ on Microsoft's Visual Studio to develop an automated software to obtain the reverse recovery parameters in LDMOS devices [7]. Their software was able to control all instruments, including applying the forward and reverse pulses, supply voltages and biasing levels, and acquiring all measurable parameters. Their system relied on an expensive Agilent 81104A pattern generator to

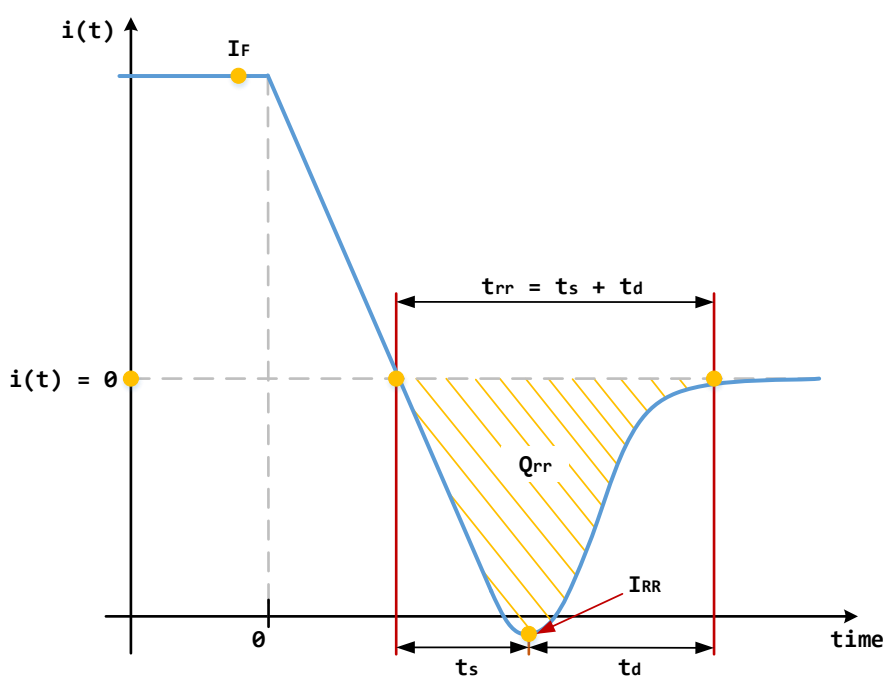

Fig. 1. Expected reverse recovery behavior 
generate the control pulses to conduct the test. A $600 \mathrm{M} \mathrm{Hz}$ LeCroy WaveSurfer 64XS and a Keithley 2612 SMU were used to provide data acquisition and supply functions. Although their setup was able to quantify all RRPs in an automated environment, the platform used for the system development was difficult to maintain as dealing with the scripts required specialized software skills. This resulted in a severe limitation for the average users of such a system, particularly with migrating patterns of the supporting software that required frequent porting to newer versions.

J. Hernández et $A l$. settled a Virtual Instrument Environment in LabView to quantify the interface state densities in MOSFETs using charge pumping techniques [8]. Although this effort was not specifically geared towards RRT characterization, it provided an important step towards enabling the usage of low-cost instrumentation to automate the RRP characterization process within a virtual instrument (VI) environment. Their development of an customizable VI for a low-cost LeCroy ArbStudio 1102 Waveform generator enabled reducing the cost of a test setup in about $30 \%$, an advantage that could be exploited in similar characterization environments.

This paper describes the development of a virtual instrument environment in LabView that allows for efficiently quantifying all RRPs in a setup amenable for usage in a production environment, providing an easy to maintain implementation and taking advantage of VI modules that allow for the usage of low-cost instrumentation. The next Section describes the elements required to perform an automated RRT, as a preamble to describe in Section III, the instrument architecture and software structure used for this work. Section IV describes the experimental design developed to assess the functionality of the test environment and the analysis of the results obtained through its application. Lastly, Section V provides some concluding remarks.

\section{Elements of An Automated Reverse Recovery TEST}

Implementing an automated virtual instrument environment (VIE) is dependent on several factors that include the development of a circuit setup to excite a device under test (DUT), an instrument architecture to provide with the signals, supply voltages, biasing, and offset levels of the test; and most importantly, the software and host platform that control the test execution. These aspects are discussed in the next sections.

\section{A. Test Circuit Setup}

The most widely accepted circuit setup for reverse recovery parameters measurement in contemporary applications is based on Joint Electron Device Engineering Council (JEDEC) standard, specifically the JESD 24-10 [9]. This circuit setup is illustrated in Figure 2. The circuit is controlled by two independent pulse sources which handle the forward and reverse voltages supplied to the DUT. When the first pulse is is applied, it biases the DUT in forward conduction. Next a second pulse is applied to bring the DUT to a reversed state, thus initiating the reverse recovery process. When the
RR current settles at zero, the test is complete and a new test cycle can be initiated.

\section{B. Instrument Architecture}

To execute a successful reverse recovery test the minimum list of instruments required include:

- Two function generators to provide forward and reverse control pulses.

- Two DC voltage sources to apply the forward and reverse voltages to the DUT.

- A bipolar voltage source for supplying power to the test setup and DUT.

- A data acquisition system capable of registering the dynamic current values circulating through the DUT during the RR process.

The pulse generators need to be accurate enough to guarantee timing the pulse widths needed for full forward condition and reverse recovery process on the DUT. Test signals in bandwidth from dozens of $\mathrm{KHz}$ up to several hundred $\mathrm{KHz}$ are typically used, and the sources must operate synchronously.

The amount of current in forward must be accurately controlled to perform a successful test. High-power devices require steady voltages reaching hundreds of volts, with currents in the order of tens of amperes. In the RR cycle, the current must be maintained steady for small values in the order of $\mu A$, and voltages in the fraction of a Volt. For this reason the DC voltage supply needs to provide an excellent regulation and a dynamic range. Finally, the data acquisition must operate in high frequencies and it should have high resolution due to the expected reverse recovery response in order of nanoseconds.

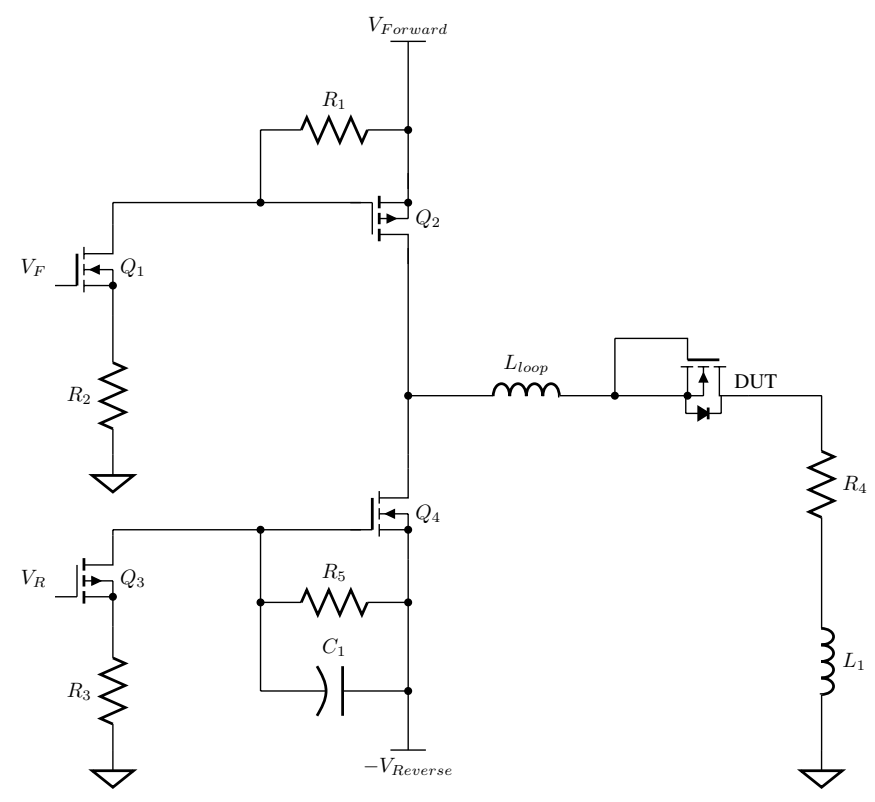

Fig. 2. RR test test by JEDEC 


\section{Software Functions}

The automation of the RRP characterization can only be achieved under software control. This software is in charge of handling all stages in the overall reverse recovery test. Configuration parameters for the test equipment must be settled first and then the communications protocol between computer and instruments needs to be established. When all instruments are ready, the test parameters need to be specified. This step allows for defining parameters such as the pulse frequency and amplitude, duty cycle, forward and reverse voltages, and window for data acquisition. Then, the test is run, where all measurable parameters are collected $\left(I_{F}, I_{r r}\right.$, $t_{s}$, and $t_{d}$ ). Finally, the extracted data is processed to quantify the non-measurable reverse recovery parameters $\left(t_{r r}, d i / d t\right.$, and $\left.Q_{r r}\right)$.

\section{Using a Virtual Instrument Setup}

An automated virtual instrument environment was developed using the software LabView ${ }^{\mathrm{TM}}$ to achieve the proposed goal. LabView ${ }^{\mathrm{TM}}$ provides a robust platform for conducting this type of measurements, while establishing a standardized programming interface compatible with many bench instruments. Moreover, its graphical user interface makes relatively simple the tasks of maintaining the software.

\section{A. Test Architecture}

In order to comply with the test requirements, a set of instruments capable of satisfying with all specifications and generating a successful signal sequence were selected.

Similar to the setup proposed by J. Rodriguez et Al. [7], the forward and reverse voltages of our setup were supplied by a Keithley 2612 Source Measurement Unit (SMU). This SMU provides the conditions to execute tests on power devices. This instrument can supply up to $200 \mathrm{VDC}$ and provide a current flow up to $10 \mathrm{~A}$. Moreover, it is sufficiently robust to guarantee stable forward current during the initial stage of the test. This instrument is handled by a GPIB communication protocol

We also adopted J. Rodriguez' et $\mathrm{Al}$. approach of using a $600 \mathrm{MHz}$ LeCroy WaveSurfer 64XS scope for data acquisition. This scope is capable of acquiring 4 Giga-samples per second, making it an excellent option to capture the RR data. This oscilloscope is controlled via a personal computer over an IP internet protocol. A current probe CPO31 fitted on one of its channels was used to capture the current flow through the DUT.

Unlike previous approaches, in our setup, control pulses were handled with a dual-channel LeCroy Arbstudio 1102 pulse generator. This instrument is a low-cost option for the test, bringing the overall test setup cost down by more than $30 \%$. Despite its low cost, it is capable of generating synchronized control pulses able to drive the reverse recovery test. The programming interface for this instruments, however, does not provide for a software interface that could be embedded into a larger virtual instrument, requiring a customized driver for its usage in this application. Such a custom VI was available through the work of J. Hernández et Al. [8]. The communication interface for this pulse generator is via a USB 2.0 port aided by .Net protocol.

Instruments in the test architecture were controlled from a host computer running the LabVIEW RR VI. The communications protocol and overall view of the test setup is shown in Fig 3.

It is important to define the proper sequence for test, as an equivocal order in the execution of the test can conduce to erroneous measurements. The sequence for our test setup is presented in Fig 4. First, the settings for pulse generator must be adjusted, followed by the application of the forward and reverse voltages to the circuit setup. Then, data starts to be captured before applying forward pulse. Then, the reverse control pulse is applied to initiate the RR process. When the reverse recovery process is finished, control pulses are disabled, and the waveforms displayed. Finally, the collected data is processed to compute the non-measurable RRPs, and lastly, all values stored in an output file.

The execution sequence was be adjusted for a LabView environment. Each stage was fixed into a frame and implemented in a structure induced by the flowchart in Fig. 4. The extracted data was processed within the LabVIEW code, requiring no ulterior steps. This consideration enables using the entire virtual instrument setup in a cyclic manner, consistent with the need of a production line environment where hundreds of devices need to be tested. Each test cycle stores the acquired and calculated values in an output file.

\section{B. Software Interface}

The developed VIE has an amenable Graphical User Interface (GUI) that allows for executing the test in an easy manner. The configuration GUI has two tabs, as illustrated in Fig. 5. The first one, designated as "Test Setup" allows for entering configuration parameters for every instrument used in the test. The top left area provides for parameters such as clock sources, sampling rates, sequencing mode, and wave indexes, among others. The middle section on the left allows for configuring the parameters for the control pulses in the function generator. Parameters configured here include frequency, amplitude, DC offset, duty cycle, and phase. The forward and reverse control pulses are configured independently. In the middle of the

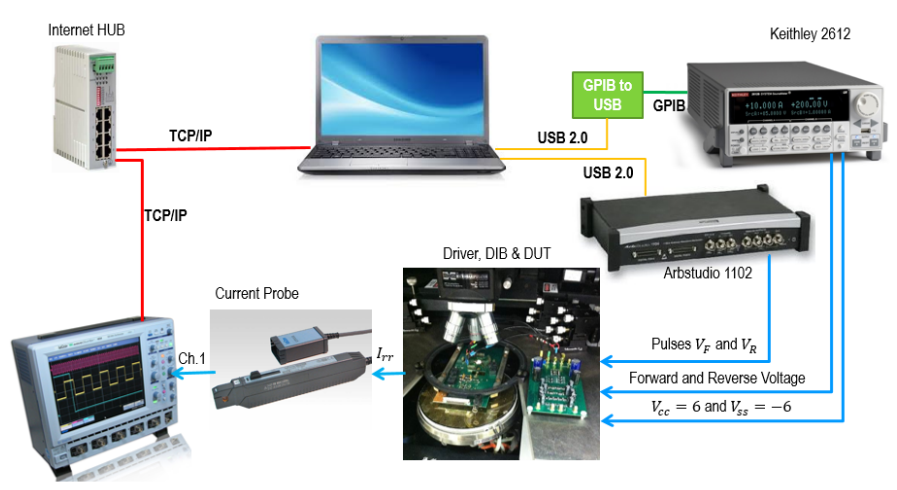

Fig. 3. Instrument communications setup 
tab, the forward and reverse voltages applied by the SMU are established. In addition, the communication protocol and current limits are defined. Finally, the right side specifies the power supply values for every element in the test circuit.

The second tab, denoted as "Test Result" provides for the collected waveforms. As shown in Fig 6, the left pane correspond to an unprocessed view of the device response. This graph usually contains high-frequency components of the measurement process. This view, although providing insight into the raw data acquired during the test, does not provide an adequate waveform for parameter quantification. For this reason we pass the signal through a smoothing filter and the resulting waveform, which depicts the central behavior of the DUT, is presented in the right of the window. Both these signals are numerically represented and stored in an output file to allow for further analysis. This test tab also provides the a summary of the conditions used for the test.

\section{Results AND AnAlysis}

To assess the functionality of our virtual environment, we performed several test on multiple LDMOS devices. All RRPs were extracted and quantified. $I_{F}$ and $I_{R R}$ were obtained from the current graph, while $t_{r r}$ is obtained from the sum of $t_{s}$

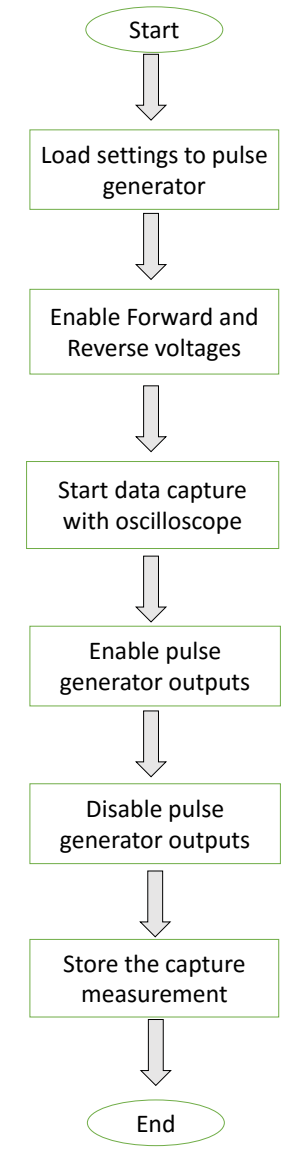

Fig. 4. Sequence for test execution

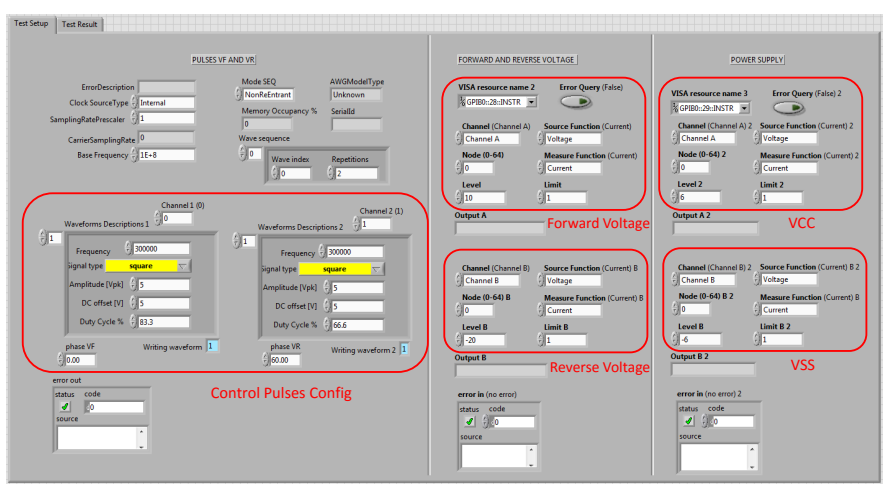

Fig. 5. Configuration parameters tab-GUI

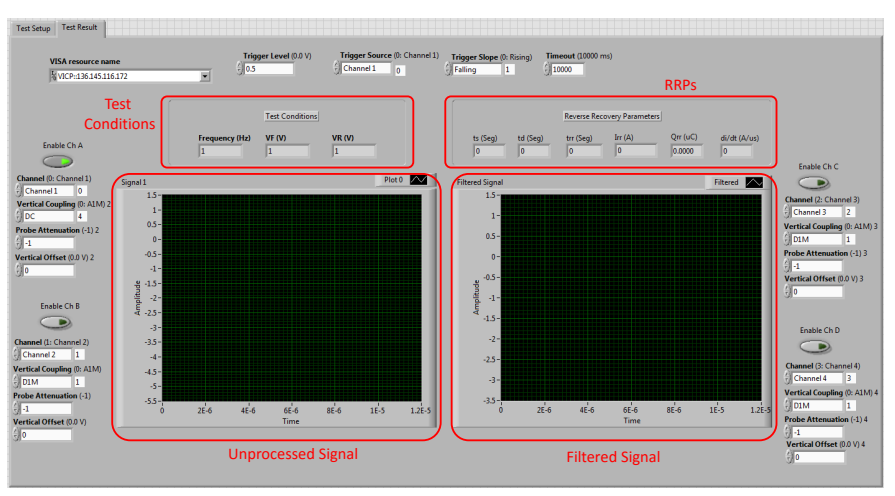

Fig. 6. Test results tab-GUI

and $t_{d}$. The switching speed $(d i / d t)$ was determined in the interval from $0.5 I_{F}$ to $0.75 I_{R R}$ as established in the JESD 2410 JEDEC standard. Finally, $Q_{r r}$ was calculated by integration of the current graph in the reverse recovery process.

To execute the experiment, forward and reverse voltages were set at $3 \mathrm{~V}$ and $-15 \mathrm{~V}$, respectively. Control pulses were settled on $300 \mathrm{KHz}, 5 \mathrm{~V}$ amplitude, $2.5 \mathrm{~V}$ offset. Moreover, the forward control pulse had $86.6 \%$ duty cycle, while the reverse control pulse had $66.6 \%$. This means that the device was forward biased for $1.24 \mu \mathrm{s}$ and reverse biased for $0.48 \mu \mathrm{s}$

To evaluate the consistency and repeatability of our VIE, three different DUTs were used to perform a test under the conditions described above. Each experiment was run five times to establish a statistically meaningful sample set. To asses the accuracy of the collected data, the average values for each DUT were compared and analyzed. Results are shown in Table I.

As indicated, the unprocessed signal contains high levels of noise as shown in Fig 7. For this reason the usage of a smoothing filter is recommended to extract the parameters in an accurate manner. We can clearly observe the reverse recovery process in the filtered signal. The parameters are quantified using the filtered data.

Finally, the data acquired is exported into a .CVS file to store the results of every single test. Fig 8 shows a test imported and graphed in Matlab. This option is useful if further 


\begin{tabular}{c|c|c|c|c|c|c|c}
\hline \multicolumn{7}{c}{ Average values for DUTs } \\
\hline \hline DUT & $I_{F}$ & $I_{r r}$ & $t_{s}$ & $t_{d}$ & $t_{r r}$ & $Q_{r r}$ & $d i / d t$ \\
\hline & $A$ & $A$ & $n s$ & $n s$ & $n s$ & $n C$ & $A / \mu s$ \\
\hline \hline 1 & 1.527 & 0.4526 & 69 & 69 & 138 & 13.2 & 8.781 \\
\hline 2 & 1.533 & 0.4724 & 75.6 & 68 & 143.6 & 14.3 & 9.068 \\
\hline 3 & 1.526 & 0.4396 & 73 & 66 & 139 & 13.3 & 8.7532 \\
\hline \hline Mean & 1.529 & 0.4548 & 72.5 & 67.7 & 140 & 13.6 & 8.8673 \\
\hline St Dev & 0.003 & 0.016 & 3E-9 & $1 \mathrm{E}-9$ & $2 \mathrm{E}-9$ & $6 \mathrm{E}-10$ & 0.174 \\
\hline \multicolumn{7}{c}{ TABLE I }
\end{tabular}

COMPARISON BETWEEN DUTS

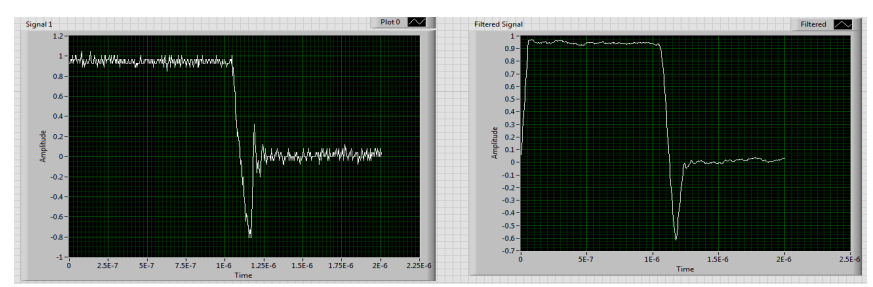

Fig. 7. Reverse recovery response

postprocessing were required.

\section{CONCLUSION}

An automated virtual instrumentation environment to measure the reverse recovery parameters of LDMOS devices was designed and presented. Previous works on automated quantification of these parameters were discussed, and opportunities for improvement were identified. The JESD 24-10 JEDEC standard was presented and briefly explained. Architecture and important considerations to perform a successful test were discussed. To provide for a suitable solution that allowed for an easily maintainable code, amenable for a production environment and low-cost testing architecture, an automated VIE design was addressed. Its GUI was shown and detailed. Finally, the proposed VIE was assessed under several conditions using different devices, demonstrating that our results were consistent and repeatable.

\section{ACKNOWLEDGMENT}

This work was supported in part by Texas Instruments, Inc. through the TI-UPRM Collaborative Program. The views presented in this paper are the opinions of the authors and

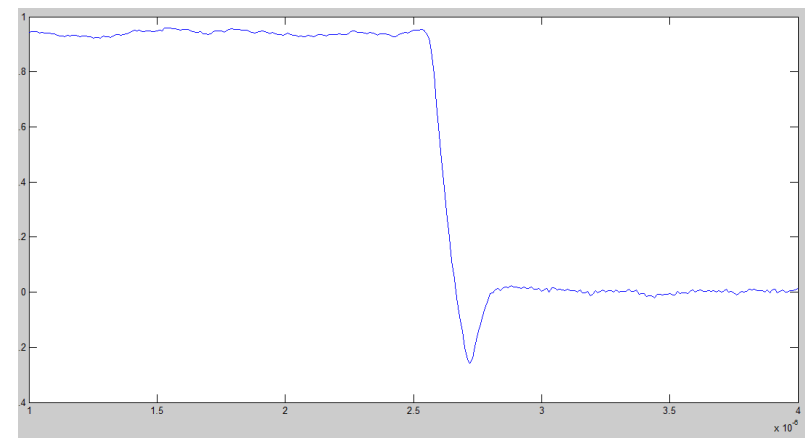

Fig. 8. Exported data in Matlab do not represent any official position of any corporation or federal agency that partially supported this work. The authors would like to thank Jose Rodriguez for his valuable help in the development of this work.

\section{REFERENCES}

[1] James D. Plummer and J.D. Meindl. A monolithic 200-v cmos analog switch. Solid-State Circuits, IEEE Journal of, 11(6):809-817, Dec 1976.

[2] S.P. Pendharkar, C.R. Winterhalter, and K. Shenai. Modelling and characterization of the reverse recovery of a high-power gaas schottky diode. Electron Devices, IEEE Transactions on, 43(5):685-690, May 1996.

[3] M.F. Catania, F. Frisina, N. Tavolo, G. Ferla, S. Coffia, and Salvatore Ugo Campisano. Optimization of the tradeoff between switching speed of the internal diode and on-resistance in gold- and platinum-implanted power metal-oxide-semiconductor devices. Electron Devices, IEEE Transactions on, 39(12):2745-2749, Dec 1992.

[4] N.Y.A. Shammas and S. Eio. A novel technique to reduce the reverse recovery charge of a power diode. In Power Electronics and Applications, 2007 European Conference on, pages 1-8, Sept 2007.

[5] F. Draghici, X. Jorda, G. Brezeanu, M. Badila, J. Millan, and P. Godignon. A system to measure reverse recovery time and stored charge at ultrafast power diodes. In Semiconductor Conference, 2001. CAS 2001 Proceedings. International, volume 2, pages 473-476 vol.2, Oct 2001.

[6] J. Stahl, D. Kuebrich, T. Duerbaum, A. Leicht, and J. Patz. A fully automated measurement set-up for the determination of the reverse recovery behaviour of ultra-fast diodes. In Power Electronics and Applications (EPE 2011), Proceedings of the 2011-14th European Conference on, pages 1-9, Aug 2011.

[7] J.A.R. Latorre, M.A. Jimenez, and R. Palomera. Automated wafer-level measurement of ldmos reverse recovery parameters. In Circuits and Systems (MWSCAS), 2012 IEEE 55th International Midwest Symposium on, pages 1072-1075, Aug 2012.

[8] J. Hernandez, J. Castrillon, M. Jimenez, A. De La Torre, P. Escalona, and R. Palomera. A virtual instrument design for low-cost charge-pumping characterization of integrated mosfets. In Test Symposium (LATS), 2015 16th Latin-American, pages 1-4, March 2015.

[9] JEDEC SOLID STATE TECHNOLOGY ASSOCIATION. Test method for measurement of reverse recovery time trr for power mosfet drainsource diodes. In JESD 24-10, Oct 2002. 